\title{
Uncertainty-Aware Model for Reliable Prediction of Sepsis in the ICU
}

\author{
Marco AF Pimentel1, Adam Mahdi, Oliver Redfern2, Mauro D Santos1, Lionel Tarassenko1 \\ 1Department of Engineering Science, University of Oxford, Oxford, UK \\ 2Nuffield Department of Clinical Neurosciences, University of Oxford, Oxford, UK
}

\begin{abstract}
Predicting the onset of sepsis from clinical data is challenging, as physiological and laboratory measurements are sampled at different frequencies and missing data are not randomly distributed. Our team ("CRASHers") propose a two-model approach, where the first predicts a probability of sepsis and the second estimates the uncertainty of these predictions. We then optimize a "decision rule" using both the probability and uncertainty to make the final prediction. A set of derived features was used to train a Gradient Boosting Machine (GBM) classification model to predict sepsis (within 6 hours). A second GBM regression model was trained to estimate the uncertainty of those predictions using a different set of derived features. Optimal hyperparameters for both models were determined using Bayesian optimisation with 5-fold cross validation (using 70\% records from each training set). The outputs from both models were then combined using logistic regression (using 15\% of records available) to re-calibrate the probability of sepsis. Due to an error in setting up the test environment for our entries, we did not obtain a valid score in the hidden test set. The combined model was evaluated on the remaining $15 \%$ of records available for training (i.e., our validation set). Our uncertainty-aware approach achieved a Utility score of 0.412 on our validation set.
\end{abstract}

\section{Introduction}

Sepsis is defined as "life-threatening organ dysfunction caused by dysregulated host response to infection". Early detection and treatment of sepsis can lead to better patient outcomes [1, 2]. With the advent of Electronic Health Record (EHR) systems, there has been an increase of studies developing prediction models to aid the identification of patients with sepsis in the intensive care unit (ICU). EHR data, however, pose a challenge to standard approaches to using machine learning to model longitudinal data.

Most data sets derived from routinely collected clinical data contain a substantial proportion of missing values and irregularly-sampled data. In particular, some laboratory tests are only performed in a subset of patients for diagnostic purposes (e.g. troponin). Furthermore, even vital signs and common laboratory tests (e.g. renal function) are measured at different intervals (which may range from 1 hour to 72 hours). The frequency of monitoring depends on internal protocols, as well as an individual patient's diagnosis and severity of illness. Most approaches for coping with these missing values or irregularly-sampled data rely on imputation methods, without accounting for the potential biases (and errors) that it may generate when making these predictions.

We propose a two-model approach, where the first model predicts a probability of sepsis and the second estimates the uncertainty of these predictions due to missing data. We then optimise a decision rule, which considers both the probability and model's uncertainty to make our final predictions.

\section{Materials and methods}

This study was performed as part of the Physionet Challenge 2019 [3].

\subsection{Dataset}

This study used two datasets provided for the Physionet Challenge 2019. These datasets were originally extracted by the Challenge's coordinators from patient admissions to three (sets A, B, C) intensive care units (ICU). All datasets contained hourly-stamped measurements for 34 distinct (time-varying) variables (8 vital signs and 26 laboratory test results), including the time (or hour) at which each value was gathered. In additions, the values of 5 demographic (static) variables are also available for each record in the datasets. Two datasets (set A and set B) with a total of 40,336 ICU patient records were made available for model development, with corresponding sepsis labels ( 1 if onset of sepsis occurs within 6 hours for patients who developed sepsis, 0 otherwise) provided for each hourlystamped measurement in each record. The third dataset (set C) was not available to the Challenge participants but was 
used to evaluate the final models during the Computing and Cardiology 2019 conference.

\subsection{Data pre-processing}

Prior to feature extraction, the distribution of each physiological measurement was manually inspected. Physiologically implausible values for certain variables are set as missing (null entries). Specifically, this process was performed for heart rate, respiratory rate, systolic and diastolic blood pressures, mean arterial pressure, temperature and pulse oximetry.

\subsection{Statistical analysis}

Our method relies on a two-model approach: (1) the first model estimates the probability of sepsis using an augmented set of features derived from the clinical data available; (2) the second model attempts to estimate the uncertainty (or error) of the predictions of the first model generated by the imputation method. For the latter, we use a second set of features that relate to the "missingness" of each time-varying variable. Both prediction and model's uncertainty are then combined to provide a re-calibrated probability of sepsis.

For the Physionet Challenge 2019, the official metric used to assess the performance of the submitted models is a customized Utility score, which rewards early prediction of sepsis (up to 12 hours before onset) and penalizes late predictions [3].

\subsection{Feature extraction}

Each patient's risk of sepsis is computed for each time point. Our model also considers the prior sequence of physiological and laboratory measurements recorded up to that timestamp. Thus, we converted each record's hourlystamped set of variables into a new set of hourly-stamped variables based on the previous and current measurements available for that record.

For the first model, we extracted a range of features from the clinical measurements, as well as deriving summary features from the time series. Static variables (e.g. age, gender), were simply repeated at each time point. For time-varying variables, we extracted the most recent measured value for each vital sign and laboratory measurement, maximum and minimum values of each vital sign within the preceding 12 and 24 hours, and difference between the two last recorded values of a subset of laboratory results (including creatinine, blood urea nitrogen, and platelet count); if no two previous values were available for a given variable, we set these variables to zero. If a given variable was completely missing for a given patient (or there were no previous or current values), the median value over the training data was imputed.
For the second model, we computed the elapsed time (in hours) since the last recorded (non-null) value for each of the 34 time-varying variables. These features capture how recently (if at all) a given variable was measured at a given timestamp. If a variable is completely missing for a given record, or there are no previous or current values, a value of 1 year (8760 hours) was imputed.

\subsection{Model description}

Both models were fit using Gradient Boosting Machines (GBMs). The GBM is an ensemble method based on using weak learners, in our case, decision trees. GBMs iteratively train collections of decisions trees to classify the training data; with each step incorporating a new decision tree, which preferentially weights the correct classification of previously misclassified training examples. We chose a GBM method on the basis of favourable comparison with other regression-based methods.

The XGBoost implementation [4] was chosen as it provides options for regularization and the handling of imbalanced classes. XGBoost also allows optimisation of other hyperparameters that control both the entire ensemble and structure of individual decision trees. To reduce overfit to the training data, we used the early stopping function, which stops the training (i.e., adding more trees) when validation scores have not improved for 50 iterations.

\subsection{Hyperparameter tuning}

Given the vast number of hyperparameter combinations to explore and their domain (i.e., the range of values that we want to evaluate for each hyperparameter), we used Bayesian optimization. Bayesian hyperparameter optimization finds the value that minimizes an objective function by building a surrogate function (probability model) based on past evaluation results of the objective. The surrogate is cheaper to optimize than the objective, so the next input values are selected by applying a criterion to the surrogate (in our case, the expected improvement was used). Bayesian methods differ from random or grid search in that they use past evaluation results to guide which values to evaluate next. A tree Parzen estimator was used as the optimization algorithm.

Hyperparameters of both models (classification and uncertainty) were tuned using 5-fold cross validation of the training set. The area under the receiver operating characteristics curve (AUROC) and the root-meansquared-error were used for the first and second model, respectively.

The range of tuned hyperparameters (same for both models) are shown in Table 1. 
Table 1. GBM's hyperparameters domain and distribution. Names of hyperparameters are those used in the XGBoost package (see [4]).

* n_estimators is fixed as it is estimated via early stopping.

\begin{tabular}{lll}
\hline Hyperparameter & Domain & Sampling \\
\hline n_estimators* & 10,000 & Fixed \\
eta & {$[0.001,1.0]$} & Log-Uniform \\
max_depth & {$[2,9]$} & Uniform \\
subsample & {$[0.4,1.0]$} & Uniform \\
colsample_bytree & {$[0.1,0.8]$} & Uniform \\
gamma & {$[0.1,5.0]$} & Uniform \\
scale_pos_weight & {$[0.2,20.0]$} & Uniform \\
lambda & {$[0.1,3.0]$} & Uniform \\
\hline
\end{tabular}

All other hyperparameters were set to their default value.

\subsection{Model development and assessment}

We evaluated the performance of the proposed prediction model by randomly dividing the 40,336 patient records into a training set (containing $70 \%$ of records from set $\mathrm{A}$, and $70 \%$ of records from set $\mathrm{B}$ ), a recalibration set (containing $15 \%$ of records from each set), and a validation set (containing the remaining $15 \%$ of records from each set).

First, we trained a GBM (binary) classification model to predict sepsis within 6 hours of a given timestamp (the "Sepsis Label") using the feature set and the best set of hyperparameters found using the hyperparameter tuning procedure described above. Secondly, we computed the negative log-likelihood for each prediction in our training set, and trained a second GBM regression model to estimate the error of those predictions using the second feature set and the best set of hyperparameters found for this second model.

Using the recalibration set, we then calculated the predicted values from both models (i.e., the probability of sepsis, and the model's uncertainty) for each record's entry, and combined both predictors into a single recalibrated score (our decision rule) with logistic regression, using "Sepsis Label" as the outcome. Finally, in order to provide a binary prediction of "sepsis", it was necessary to threshold the score value (a probability between 0 and 1). A threshold was chosen that maximized the Utility score on the recalibration set.

We compared the performance between the proposed uncertainty-aware two-model approach; the single GBM classification model (which threshold was re-calculated using the same methodology); and the baseline model supplied by the challenge, on the held-out validation set (which was not used at any point during the development of the model presented in this study).

\section{Results}

The evaluation metrics on the whole held-out validation set (containing 6,051 records from set A and set B) for each model are shown in Table 2. Evaluation metrics include the AUROC, the area under the precision-recall curve (AUPRC), the F1-score, the accuracy and the Utility score.

Table 2. Evaluation metrics of the uncertainty-aware model (our two-model approach, M2), the single GBM model that does not include the estimations of model uncertainty (single-model approach, M1), and the baseline model based on a time-to-event regression model (B0).

\begin{tabular}{llll}
\hline Metric & B0 & M1 & M2 \\
\hline AUROC & 0.702 & 0.829 & $\mathbf{0 . 8 4 1}$ \\
AUPRC & 0.057 & 0.101 & $\mathbf{0 . 1 1 2}$ \\
Utility score & 0.186 & 0.399 & $\mathbf{0 . 4 1 2}$ \\
\hline
\end{tabular}

Our uncertainty-aware approach (M2) achieved an AUROC of 0.841 and a Utility score of 0.412 . The Utility score of our model is substantially higher than the baseline model (Utility score of 0.186 ) when evaluated on the heldout validation set. We also note an improvement on the Utility score of the uncertainty-aware predictions with respect to the predictions that do not take into account our estimation of uncertainty.

Table 3 shows the AUROC and Utility score values obtained for each validation set (sets A and B). We note that the uncertainty-aware model provided a larger performance improvement (as given by the Utility score) in test set B. Figure 1 shows the distribution of the uncertainty estimations in the records with positive cases of sepsis in the validation set.

We note that the held-out validation set used to report these results was generated from the publicly available training sets. We were not able to obtain a valid score in the hidden test set due to an error in setting up the test environment for the entries.

\section{Discussion and conclusions}

At the thresholds (or operating points) found for each model, we observed a large improvement in the Utility score with our uncertainty-aware model (M2) relative to the baseline model B0 (0.412 vs. 0.186), and a smaller, yet substantial, improvement relative to the single model (M1), which does not include the uncertainty estimates (0.412 vs. 0.399). The improvement in performance of model M2 is most notable for test set B (Table 3).

An important goal that we aimed to achieve with modelling the uncertainty of the GBM prediction model was increasing the reliability of predictions. Prediction reliability is orthogonal to prediction accuracy, and another 
Table 3. Evaluation metrics of the uncertainty-aware model (our two-model approach, M2), the single GBM model that does not include the estimations of model uncertainty (single-model approach, M1), and the baseline model based on a time-to-event regression model (B0) for both test sets $\mathrm{A}$ and $\mathrm{B}$.

\begin{tabular}{llll}
\hline Metric & B0 & M1 & M2 \\
\hline AUROC & & & \\
Validation set A & 0.703 & 0.826 & $\mathbf{0 . 8 3 5}$ \\
$\quad$ Validation set B & 0.690 & 0.846 & $\mathbf{0 . 8 6 0}$ \\
Utility score & & & \\
Validation set A & 0.221 & 0.424 & $\mathbf{0 . 4 2 6}$ \\
$\quad$ Validation set B & 0.096 & 0.353 & $\mathbf{0 . 3 8 3}$ \\
\hline
\end{tabular}

study [5] showed that state-of-the-art machine learning models are often not reliable - they are not well-calibrated to correlate model confidence with model strength. Imputation methods for coping with missing data and/or irregularly-sampled sequences may contribute for the lack of reliability of model predictions. Thus, we evaluated our uncertainty calibrated model (M2) against the GBM model with no uncertainty recalibration (M1), and the results in Table 2 and Table 3 show that, although the improvement in discrimination (as given by the AUC) is relatively modest (0.835 vs. 0.826$)$, the improvement of the Utility score is substantial (0.412 vs. 0.399). Hence, the predictions from the uncertainty-aware model appear to

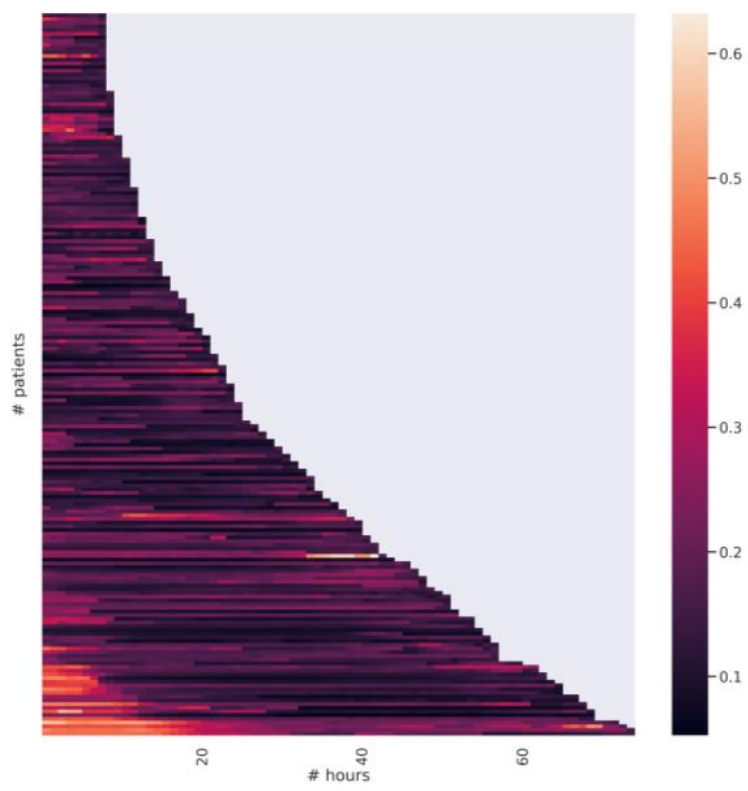

Figure 1. Stacked timelines of estimated uncertainty for the patients who developed sepsis (positive cases) in test set. Darker colors correspond to lower values of uncertainty. Sequences are ordered by sequence/record length (records with a length over 75 hours are not shown). We see that the uncertainty is generally larger at the start of each sequence. provide better calibration.

We proposed an uncertainty-aware approach that has the potential to enhance reliability of both interpretations and predictions of sepsis provided by a GBM model. Further analysis of prediction reliability may be necessary in order to demonstrate that the model is accurately calibrated and thus can defer predictions when making prediction with an "I don't know" option. This option could enhance/aid interpretability if prediction models are incorporated into clinical decision-making tools.

\section{References}

[1] M. Singer et al., "The Third International Consensus Definitions for Sepsis and Septic Shock (Sepsis-3)", JAMA, vol. 315, no. 8, pp. 801-810, Feb. 2016.

[2] C. W. Seymour et al., "Time to Treatment and Mortality during Mandated Emergency Care for Sepsis", $N$ Engl J Med, vol. 376, no. 23, pp. 2235-2244, Jun. 2017.

[3] M. A. Reyna, C. Josef, R. Jeter, S. P. Shashikumar, M. B. Westover, S. Nemati, G. D. Clifford, A. Sharma, "Early prediction of sepsis from clinical data: the PhysioNet/Computing in Cardiology Challenge 2019", Critical Care Medicine 2019, In press.

[4] T. Chen, and C. Guestrin, "XGBoost: A scalable tree boosting system", ACM SIGKDD International Conference on Knowledge Discovery and Data Mining, Aug. 2016.

[5] M. P. Naeini, G. F. Cooper, and M. Hauskrecht, "Obtaining well calibrated probabilities using Bayesian binning", $A A A I$, Jan. 2015.

Team Name: CRASHers

Address for correspondence:

Marco AF Pimentel

Institute of Biomedical Engineering

Department of Engineering Science

University of Oxford

Old Road Campus Research Building, Roosevelt Dr

Oxford OX3 7DQ, UK

E-mail:marco.pimentel@eng.ox.ac.uk 